\title{
Potential Use of Ultrasonic Cavitation Threshold to Non-Invasively Differentiate Cystic Masses
}

\author{
Brian E. O’Neill*, Ellen Chang, Nam Yu \\ Department of Translational Imaging, Houston Methodist Research Institute, Houston, USA \\ Email: BEEOneill@HoustonMethodist.org
}

Received 11 October 2014; revised 8 November 2014; accepted 2 December 2014

Copyright (C) 2014 by authors and Scientific Research Publishing Inc.

This work is licensed under the Creative Commons Attribution International License (CC BY). http://creativecommons.org/licenses/by/4.0/

(c)

\begin{abstract}
Objectives: To demonstrate in vitro that changes in ultrasound cavitation threshold might be used for non-invasively distinguishing high viscosity mucinous fluid from low viscosity serous fluid in cystic masses, based on the facts that cavitation threshold increases with increasing viscosity and that cavitation microbubbles are observable with diagnostic ultrasound. Methods: An in vitro model of a cyst was designed using dilutions of ultrasonic gel, and the cavitation threshold of this model was determined using focused and unfocused ultrasound for bubble initiation and clinical ultrasound b-scan for detection. Results: Viscosities of dilutions between $0 \%$ and $30 \%$ gel were had viscosities measuring between $1.05 \pm 0.08 \mathrm{cP}$ and $6600 \pm 875 \mathrm{cP}$. Inertial cavitation in the latter was determined to require an order of magnitude greater intensity, at $1 \mathrm{MHz}$ and $100 \%$ duty cycle, than the former $\left(>2.2 \mathrm{~W} / \mathrm{cm}^{2} \mathrm{vs}\right.$. $\left.<0.19 \mathrm{~W} / \mathrm{cm}^{2}\right)$ using unfocused ultrasound. A four-fold increase in the peak negative pressure was required to initiate significant bubble activity using $1.1 \mathrm{MHz}$ and $50 \%$ duty cycle focused ultrasound in the $6600 \mathrm{cP}$ fluid compared with the $1 \mathrm{cP}$ fluid. Based on these results, it was estimated that a threshold could be defined that would result in no bubbles in $\mathbf{9 9 . 9 \%}$ of mucinous cysts and just $22 \%$ of serous cysts. The remaining $\mathbf{7 8 \%}$ of patients presenting with serous cysts would be positively identified by detection of bubbles, and would be spared an unnecessary biopsy. Conclusions: The cavitation threshold may be used non-invasively to distinguish between high viscosity and low viscosity fluids in cysts and reduce biopsies on serous cysts.
\end{abstract}

\section{Keywords}

Fluid Viscosity, Cavitation Threshold, Mucinous Cyst, Serous Cysts

\footnotetext{
${ }^{*}$ Corresponding author.
} 


\section{Introduction}

Fluid containing masses in the body often pose a diagnostic dilemma on conventional imaging which often shows only nonspecific features. Pancreatic cysts are usually categorized as serous or mucinous, and this distinction has management and prognostic implications since only the latter has malignant potential [1] [2]. To differentiate between these two, fine needle aspiration under endoscopic ultrasound guidance is often required, since non-invasive modalities (ultrasound, CT and MRI) are unable to distinguish between these two with great certainty [3]. The major physical difference between the two fluids is viscosity. Serous (serum-like) fluids, such as blood serum, have viscosities around $1.4-1.8 \mathrm{cP}$. The condition known as hyperviscosity, where blood is too thick, still has serum viscosity numbers in low single digits between 3 and $5 \mathrm{cP}$ [4] [5]. On the other hand, various mucinous fluids measured in the literature all have typical viscosities 20,000 to $140,000 \mathrm{cP}$ [6]-[8], 4 to 5 orders of magnitude greater than serous fluids. It clearly should be possible to differentiate these two conditions on this basis. Differentiation of other types of viscous fluids, such as in endometrial cysts or infected wounds, may be similarly possible. However, this may depend largely on the highly variable particulars of the fluid mixtures that strongly impact on the viscosity.

Various techniques have been devised to detect viscosity differences in cysts ultrasonically with limited success. A combination of Doppler ultrasound and ultrasonic radiation force proved somewhat successful in that if a signal could be obtained at all, low viscosity fluid could be confirmed [9]-[11]. However, a large part of the problem lies in the lack of signal from certain of these mostly anechoic pockets and inconsistency in either radiation forces applied or the acoustic streaming resulted in, which is boundary condition dependent for small volumes. A large scale multicenter trial found that this technique could not reliably distinguish between ostensibly highly viscous endometriomas and other lesions because of these effects [12]. If it were possible to introduce microbubbles directly into the cysts, for example and observe their motion under the radiation force, this would likely easily differentiate one type from the other; however, introducing contrast agents is no less invasive than the needle aspiration.

An alternative is to generate microbubbles endogenously and analyze their motion. As it happens, the threshold for inertial cavitation itself is quite sensitive to changes in viscosity [13] [14]. The presence of microbubbles is then easily detected with conventional ultrasound imaging. In this study, we seek to establish whether this might be a useful technique for testing mucinous vs. serous cysts by developing a cyst model with controlled viscosity, producing bubbles endogenously with ultrasound-guided high intensity ultrasound at increasing power, and detecting these bubbles with ultrasound imaging.

\section{Materials and Methods}

\subsection{Model of Cystic Lesions}

A model of viscous cystic lesions was developed based on ultrasound gel (Aquasonic Clear, Parker Laboratories, Inc., Fairfield, NJ, USA) diluted with water. We generally used five levels of dilution, $0 \%, 5 \%, 10 \%, 20 \%$ and $30 \%$ gel. At each dilution, 5 samples were made for testing with focused ultrasound, and one each for unfocused ultrasound and for measuring viscosity. Dilutions were by mass, that is, $1 \mathrm{~g}$ of gel and $4 \mathrm{~g}$ of water is $20 \%$ gel. This is done because it is simpler and more accurate to measure weight than volume, given the possible inclusion of air in the gel. Dilute gel samples were well mixed by vortexing for 5 minutes, then spun at $250 \mathrm{~g}$ in a centrifuge (Sorvall Legend XTR, Thermo Fisher Scientific, Inc., Waltham, MA, USA) for a minimum of 10 minutes to clear as much trapped air as possible. The gel was then injected into a latex balloon, which was tied shut and centrifuged again. It was important to verify that as little air as possible was trapped along with the gel. These model "cysts" were then placed in a holder in a bath (see Figure 1) for focused ultrasound treatment or coupled with $100 \%$ gel directly on the surface of the physiotherapy device for unfocused ultrasound treatment.

Viscosity of the gel samples were measured approximately using a relatively simple device (Figure 2), based on measuring the pressure required to force the sample through tubing of various lengths and diameters at constant velocity using a syringe pump (Harvard Pump 11 Plus, Harvard Apparatus, Holliston, MA, USA). Although this system is not as accurate as many commercial systems, it has the advantage of covering a very wide range of viscosities very cheaply with reasonable amounts of sample $(<5 \mathrm{ml})$.

\subsection{Sonication}

A Philips Research Unison Therapy and Imaging Probe System (“TIPS”, Philips Research, Inc., Briarcliff Manor, 


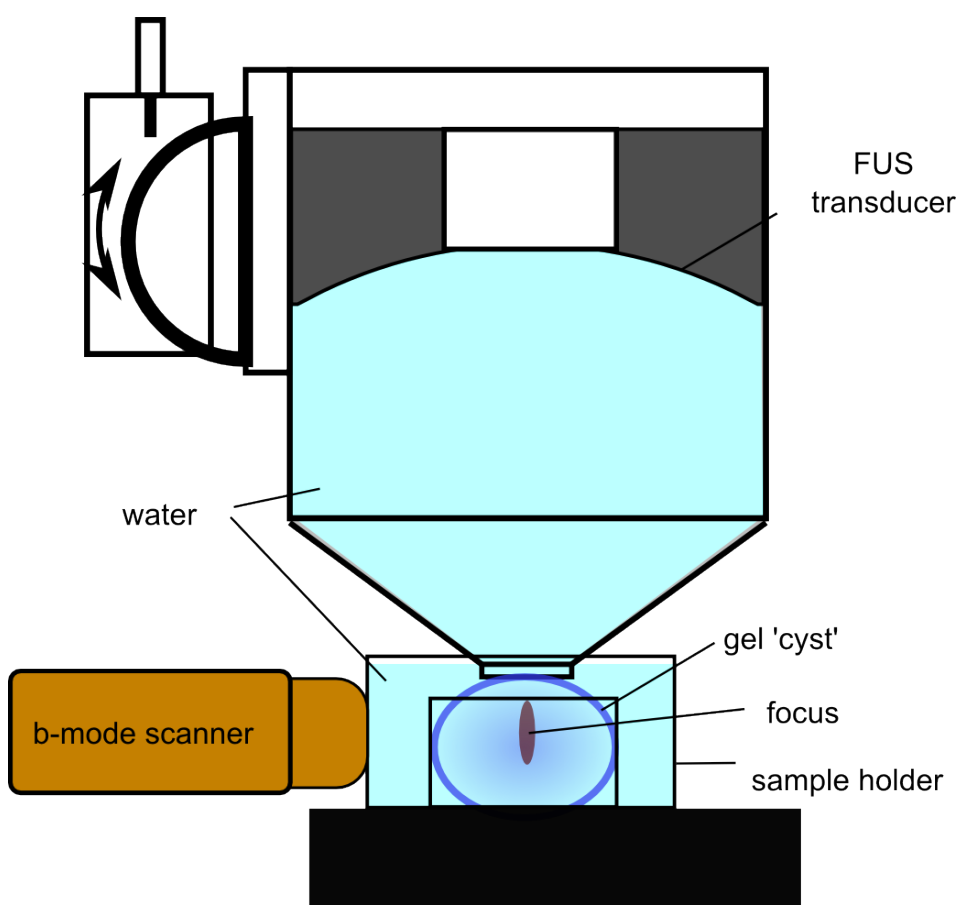

Figure 1. Setup for treating model cyst using the Philips Unison "TIPS" system. The "cyst" consists of a latex balloon filled with varying dilutions of ultrasound gel and water.

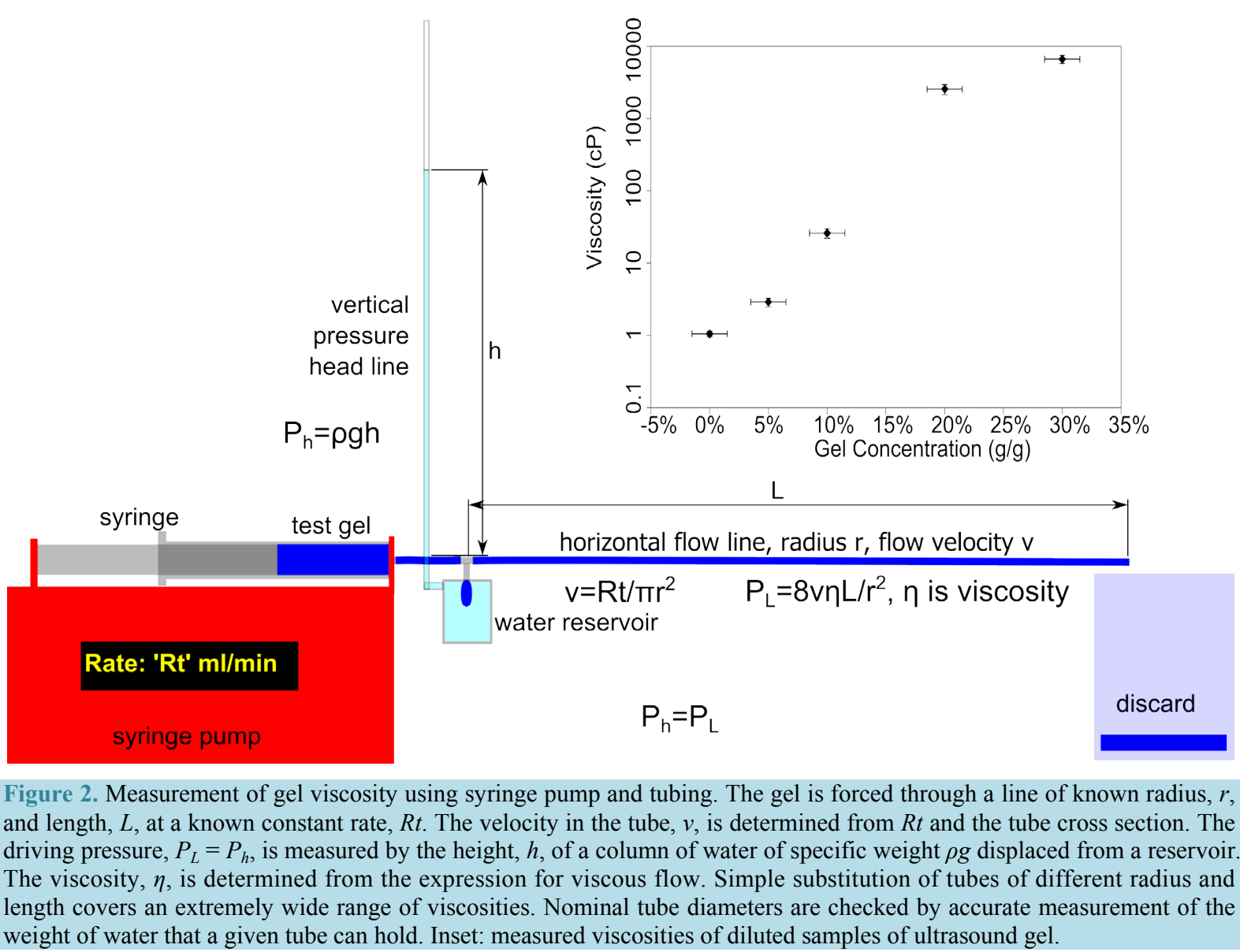


NY, USA) was used to direct focused ultrasound into the "cyst". This focused ultrasound surgery (FUS) system directs high intensity focused ultrasound, produced at $1.1 \mathrm{MHz}$ center frequency, from a spherically focused transducer into a focal spot approximately $1.5 \mathrm{~mm}$ in diameter and $7 \mathrm{~mm}$ in length located $8 \mathrm{~cm}$ in front of the transducer. The output of the transducer may be controlled up to $40 \mathrm{~W}$ total acoustic power with complete control over pulse length and duty cycle (d.c.). The holder containing the sample "cyst" was filled with degassed water and coupled to the transducer through the TIPS degassed water column (Figure 1). The TIPS system was aligned using melting of a plastic absorbing target to locate the focal spot just outside of and centered on the acoustic window, $8.0 \mathrm{~cm}$ from the transducer. The transducer head was then lowered so that the acoustic window just came into contact with the top of the "cyst" sample. From the side, a medical ultrasonic scanner with 6 MHz abdominal probe (Acuson Sequoia 512, Siemens Healthcare, Malvern, PA, USA) imaged horizontally through the plane of focus at the center of the sample. For each sample, the power of the TIPS transducer at 1.1 $\mathrm{MHz}$ was ramped from low to high, using a duty cycle of $50 \%$, duty cycle (d.c.) of $1 \mathrm{~Hz}$, for 5 pulses at each power. During the 5 seconds of treatment, the imaging system captured the data in a DICOM cine movie. The same approach was used with unfocused ultrasound, using a $1 \mathrm{MHz}$ plane transducer (Metron Accusonic). The therapy transducer was stepped from $0.2 \mathrm{MPa}$ to $2.4 \mathrm{MPa}$ nominal pressure at $100 \%$ d.c. At each step, 5 seconds of imaging data was captured with the Siemens system at $15 \mathrm{MHz}$.

\subsection{Analysis}

For both test methods, the movies were analyzed offline using ImageJ software (National Institutes of Health, Bethesda, MD, USA). The sudden appearance of increased backscatter from newly produced microbubbles was used as indication of the cavitation threshold. For the unfocused ultrasound, this was clear enough to easily distinguish the onset of cavitation. For the focused ultrasound results, a circular region of interest (ROI) 112 pixel widths in diameter (area is 9863 pixels) was drawn over the center of the "cyst" and the signal inside was thresholded at 95 of 256 grey levels to find the signal from the bubbles. This threshold was arbitrarily chosen in the range well above the noise in the ROI but below almost all signal attributed to bubbles, and was constant across all viscosities and experimental runs. The total number of pixels, $A_{b}$, in the ROI with signal intensity above the threshold was used as a measure of gas bubble activity.

The dependence of this area on the viscosity of the fluid and the amplitude of the applied focused ultrasound was further analyzed. The ability to distinguish between low viscosity water and high viscosity $30 \%$ gel with a single test was analyzed by considering overlap of the probability densities assuming normal and log-normal distributions. Using this data and R [15], we are able to estimate the likely incidence of false positives and false negatives in a test for a malignant cystic neoplasm based on selecting some level of $A_{b}$ as the cutoff for a diagnosis. For example, the test might assume that, with a $3 \mathrm{MPa}$ focused ultrasound application, an observed $\mathrm{A}_{\mathrm{b}}$ above the cutoff would indicate bubble activity and therefore a low likelihood of high viscosity (mucinous) fluid. This would be a negative diagnosis. $A_{b}$ below the cutoff would indicate no bubble activity, corresponding to a high likelihood of mucinous fluid, a positive diagnosis. Since it is critical to maximize the sensitivity to viscosities greater than $6000 \mathrm{cP}$ and minimize the number of false negatives, the cutoff should be on the high end of the $30 \%$ gel distribution. We considered the $0.95,0.98,0.99,0.999$ quantiles as possible cutoffs (the fraction of false negatives will be 1 -quantile ). The corresponding $A_{b}$ is estimated from the $30 \%$ gel data using quantile in $\mathrm{R}$, which is based on [16]. The probability of obtaining an $A_{b}$ less than this in a low viscosity water sample (a false positive) was then calculated, based on fitting a log-normal distribution to the water data.

\section{Results}

\subsection{Viscosity of Gel Samples}

The viscosity of the gel samples covered a wide range. Our laboratory viscometer yielded the accepted viscosity of water to less than $5 \%$, with a calculated measurement error of less than $10 \%$. Although the measurement error increased with increasing viscosity of the samples, the device performed well in all orders of magnitude, giving consistent results when using tubes of different diameters and lengths, and over a range of fluid velocities (data not shown). Figure 2 and Table 1 give the measured viscosities for the relevant gel samples. A clear jump in viscosity of almost 2 orders of magnitude occurs between the $10 \%$ and the $20 \%$ dilutions. Concentrations above the $30 \%$ gel were not considered because they proved excessively difficult to degas and force through a standard syringe. 


\subsection{Cavitation Threshold Using Planar Ultrasound}

When the cavitation threshold is breached, a noticeable and immediate jump in scattered ultrasound occurs in the detecting ultrasound signal (Figure 3). This breach may take some time to occur, even up to a several tens of seconds after the therapeutic ultrasound is turned on in the $20 \%, 2500 \mathrm{cP}$ sample. When the signal is turned off, the bubbles remain clearly visible. Those in low viscosity media (water, $5 \%$ gel) continue to move well after the

Table 1. Measured viscosity of gel dilutions. The third column gives the uncertainty estimated from uncertainties in the measured variables $h, L, r$ using standard error propagation methods.

\begin{tabular}{ccccc}
\hline Sample & Viscosity $(\mathrm{cP})$ & Uncertainty $(\mathrm{cP})$ & Std. Dev. $(\mathrm{cP})$ & $\mathrm{N}$ \\
\hline $0 \% \mathrm{Gel}$ (water) & 1.05 & 0.0882 & 0.0881 & 14 \\
$5 \% \mathrm{Gel}$ & 2.9 & 0.376 & 0.229 & 6 \\
$10 \% \mathrm{Gel}$ & 26 & 3.88 & 6.11 & 8 \\
$20 \% \mathrm{Gel}$ & 2500 & 390 & 550 & 9 \\
$20 \% \mathrm{Gel}$ & 6600 & 875 & 496 & 5 \\
\hline
\end{tabular}
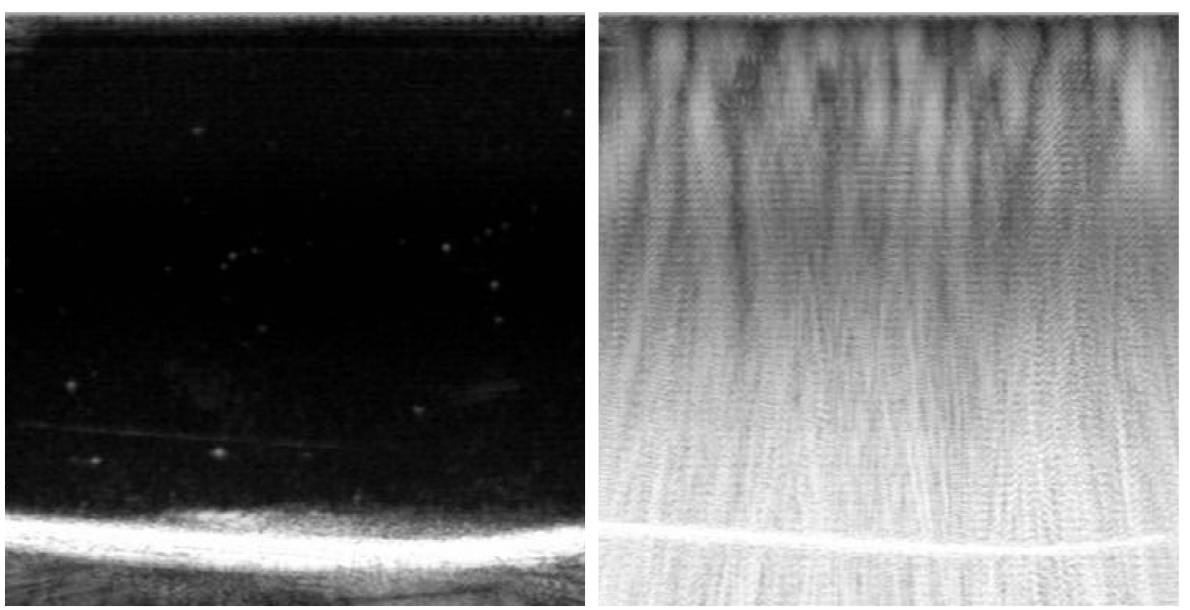

A

B

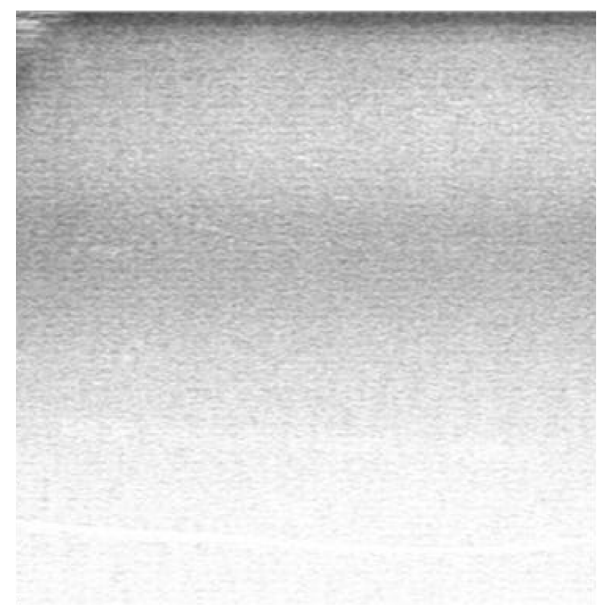

C.

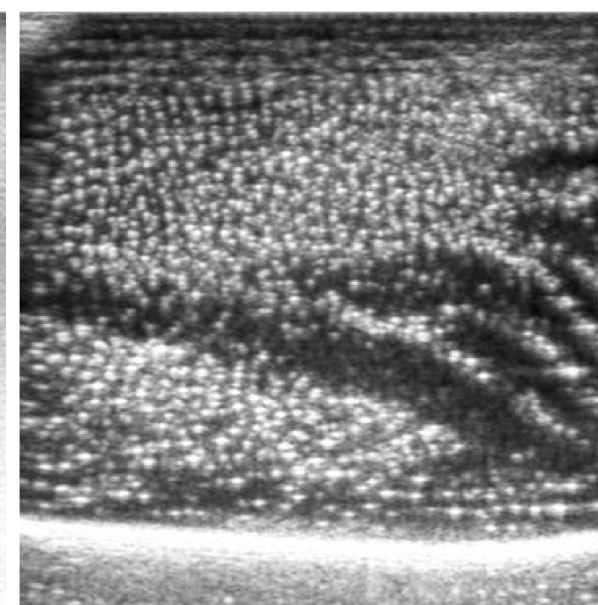

D

$5 \mathrm{~mm}$

Figure 3. Images obtained using $14 \mathrm{MHz}$ contrast enhanced imaging during unfocused treatment of $10 \%$ gel. The magnification for all frames is indicated by the scale bar in (D). Prior to sonication, gel is mainly clear (A). When the high power ultrasound is initially turned on, strong signal is observed from the ultrasound scattered from container walls (B). After a few seconds, the scatter increases dramatically and becomes less coherent (C). After turning off the high power ultrasound, imaging reveals a large number of bubbles remaining in the gel (D). 
ultrasound is turned off and begin to rise to the surface, while those in viscous media $(10 \%, 20 \%$ gel $)$ almost immediately stop moving and remain fixed, although they will dissipate over $24 \mathrm{hrs}$. The cavitation thresholds observed when treating the gels with the planar transducer are shown as a function of gel viscosity in Figure 4, with error bars indicating uncertainty in the nominal transducer power. The gray regions indicate the limits of transducer output. The threshold appears to increase almost linearly with the log of viscosity. In general, reducing the duty cycle resulted in higher cavitation thresholds. For this reason, all data shown from this unfocused transducer are at $100 \%$ duty cycle, which allowed successful interrogation of most of the samples. Even so, the $30 \%$ gel cavitation threshold was not breached by the transducer device, which has a maximum nominal output of $2.4 \mathrm{~W} / \mathrm{cm}^{2}\left(2.2 \pm 0.1 \mathrm{~W} / \mathrm{cm}^{2}\right.$ calibrated intensity). In contrast, both the water and the $5 \%$ gel solution were immediately cavitating at the lowest power setting of the system, $0.19 \pm 0.01 \mathrm{~W} / \mathrm{cm}^{2}$ calibrated intensity.

\subsection{Appearance of Cavitation Using Focused Ultrasound}

With focused ultrasound, the acoustic pressure is greatest at a single location, and most often this is where the bubble will first form. Strong scatter is immediately visible from this region, and remains after the driving ultrasound is turned off. Again, with low viscosity solution, bubbles will visibly move after the focused ultrasound is turned off while for high viscosities, they will quickly come to a stop. In Figure 5, the images show the typical appearance of cavitation bubbles in the phantom, while the graph gives mean cross sectional area $\left(\mathrm{A}_{\mathrm{b}}\right.$, in pixels) of the bubble signal as a function of acoustic power for gels of different viscosities. Figure 6 shows the distribution of $\mathrm{A}_{\mathrm{b}}$ for the different dilutions at an acoustic pressure of $3 \mathrm{MPa}$ to illustrate how one might distinguish high viscosity fluids ( $20 \%$ and $30 \%$, similar to mucinous fluid) from low viscosity fluids $(0 \%$ and $10 \%$, similar to serous fluid) using this method. Because cavitation is a stochastic process, the variability is significant. $\mathrm{P}$-values are relative to water.

Based on these results, Table 2 gives the likely incidence of false negatives (incorrect diagnosis of non-mucinous cyst) and false positives (incorrect diagnosis of mucinous cyst) if mucinous cysts are assumed to behave like the $30 \%$ gel and serous cysts like water, for various cutoff values of $A_{b}$. For $A_{b}$ of 171 pixels, the incidence

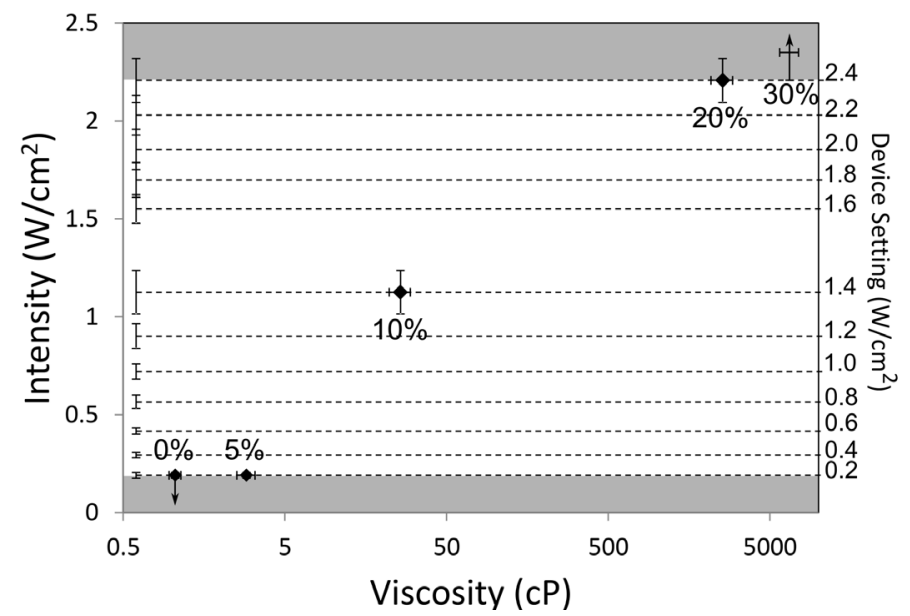

Figure 4. Minimum ultrasound intensity at which cavitation was induced in model "cysts" containing gel of varying viscosities. The ultrasound intensity was produced using a planar physiotherapy device (Metron Accusonic M970, Patterson Medical, Bolingbrook, IL, USA) driven at $100 \%$ duty cycle and calibrated using the radiation force method. Possible device settings are indicated by dotted lines with error bars on the left while the gray bands indicate lower and upper limits on its output. Both $0 \%$ gel $(1 \mathrm{cP})$ and $5 \%$ gel $(2.9 \mathrm{cP})$ samples were cavitating at the lowest intensity available, $0.19 \pm 0.01 \mathrm{~W} / \mathrm{cm}^{2}$. The arrow indicates the possibility that the actual threshold is below the minimum available intensity. The $30 \%$ gel $(6600 \mathrm{cP})$ did not cavitate even at the highest intensity of $2.2 \pm 0.1 \mathrm{~W} / \mathrm{cm}^{2}$, as indicated by the arrow pointing beyond the maximum available intensity. 


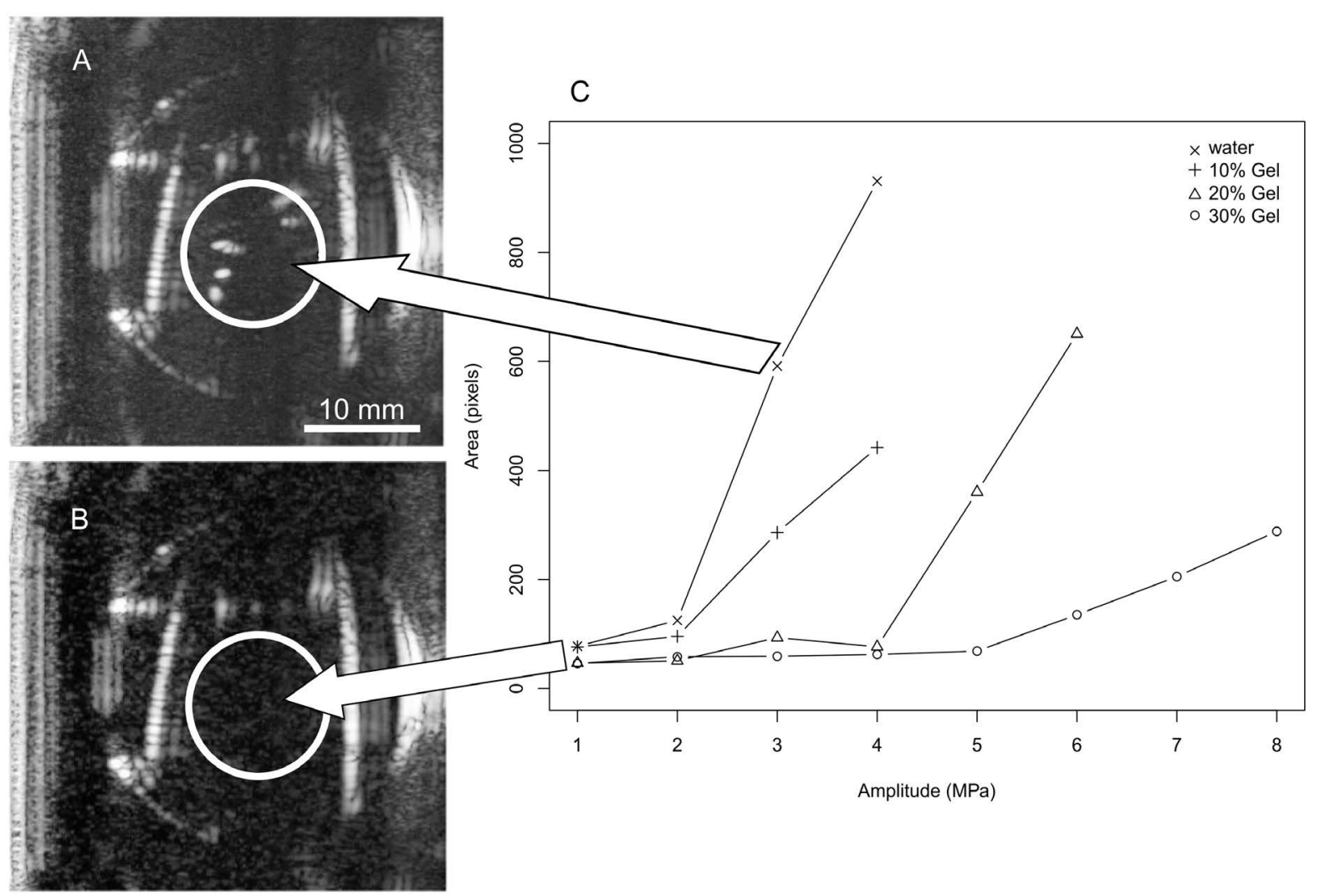

Figure 5. Bubble detection cross-sections imaged at $6 \mathrm{MHz}$ during irradiation of model cysts with focused ultrasound, showing bubble activity (A) and no bubble activity (B) in the ROI (white circle). Echogenic cross sectional area in the ROI is measured and plotted $(\mathrm{C})$ as a function of peak negative pressure and gel dilution. Other exposure parameters were $50 \%$ duty cycle, $1 \mathrm{~Hz}$ repetition rate and focused ultrasound frequency of $1.1 \mathrm{MHz}$.

of false negative will be $<1 / 1000$, while the incidence of false positive is about $22 \%$ ( $99.9 \%$ sensitivity and $78 \%$ specificity), as indicated by the horizontal line in Figure 6 . Thus $78 \%$ of patients with serous cysts could be spared a needless biopsy using this method, with very reasonable probability of detecting mucinous cysts.

\section{Discussion}

Fluid containing masses are frequently encountered in clinical practice, and the ability to non-invasively differentiate the type of fluid may be helpful in many scenarios. Pancreatic cystic neoplasms are typical examples of this. Serous cystic tumors containing thin fluid are benign and can be managed by observation, while mucinous cystic tumors containing viscous fluid have malignant potential and generally require surgical management. In one study of fluid samples from pancreatic cysts, quantitative viscometry could identify mucinous tumors from benign serous cystic masses or inflammatory pseudocysts with $89 \%$ sensitivity and $100 \%$ specificity using a viscosity threshold slightly above that of serum [17]. In fact, this distinction is typically obvious on gross inspection of the fluid, and the so called "string sign"-in which a drop of the fluid is placed between the thumb and index finger and stretched into a string of varying lengths - has been advocated as a simple and useful test of "thickness" that can identify mucinous tumors [18]. Likewise, differentiation of endometriomas, which contain thick bloody material and therefore are referred to as "chocolate cysts," from other gynecological cysts, is clinically relevant. Yet another more frequently encountered clinical dilemma is whether a postoperative fluid collection is an abscess (filled with thick pus) requiring drainage or a non-infected seroma.

While there are clues to differentiate these conditions with conventional imaging techniques, an invasive diagnostic approach is often required to obtain fluid samples. Diagnostic ultrasound is often used as an imaging tool to assess pancreatic cysts and guide biopsies, but it is not sensitive to viscosity. We have developed a robust method for determining viscosity based on changes in cavitation threshold. All other things being equal, the 


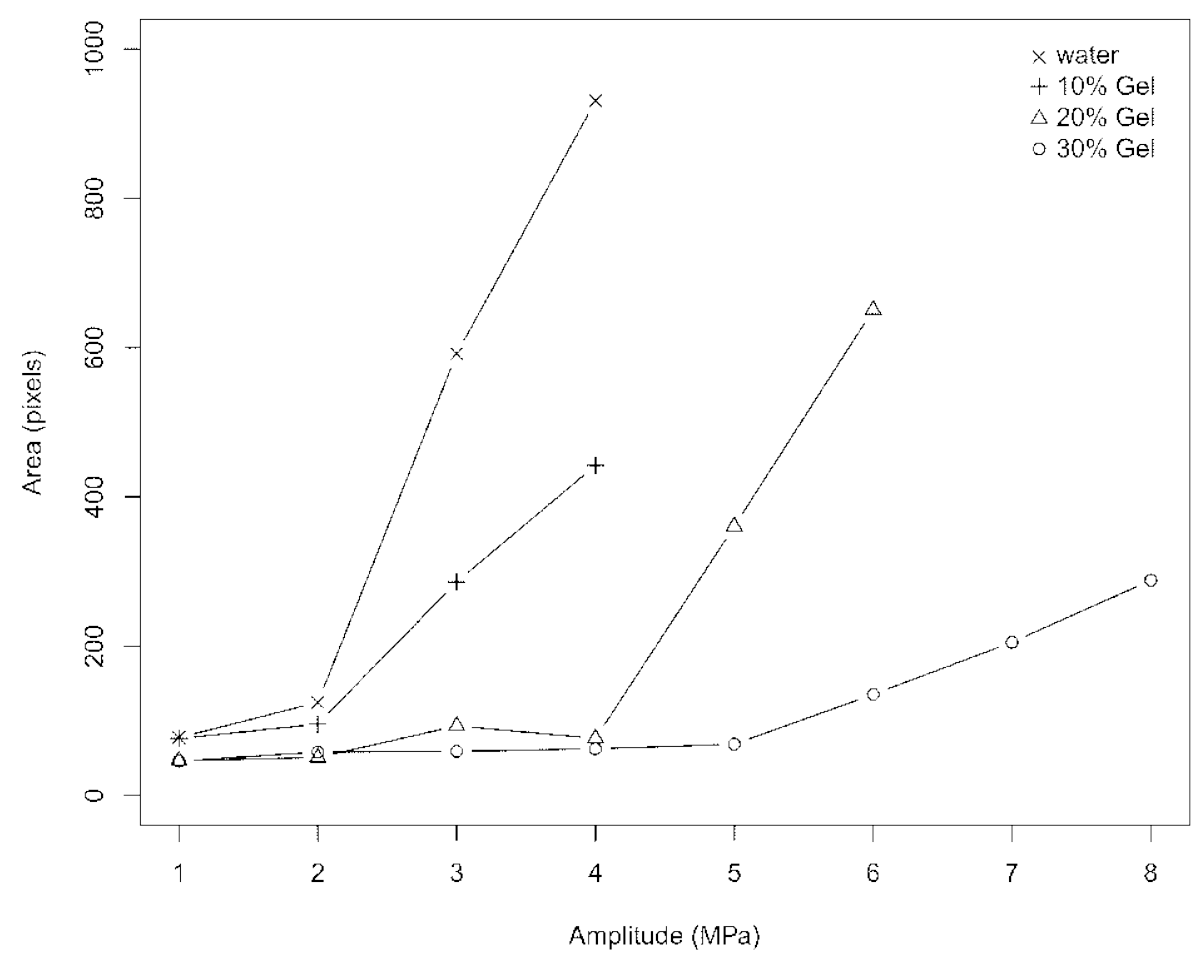

Figure 6. Distributions of bubble cross sections of various gel samples at $3 \mathrm{MPa}$. Boxplots indicate medians with upper and lower hinges (25th \& 75 th percentiles). P-values are calculated relative to the water sample using ANOVA. Although the means and medians are significantly different between distributions, the overlap is not negligible. Dotted line indicates level of $99.9 \%$ sensitivity for the $30 \%$ gel. If bubbles are observed $\left(A_{b}\right.$ is above this line), we can be very confident that the sample consists of a low viscosity fluid. If bubbles are not observed ( $A_{b}$ is below this line), a low viscosity sample is unlikely, but not impossible. This uncertainty can be easily managed by appropriate further testing (biopsies).

Table 2. Projected errors in "mucinous cyst" diagnosis based on lack of bubble signal. $A_{b}$ is the cutoff threshold (minimum size of "bubble" activity), in pixels, detected within a ROI of total area 9863 pixels using an arbitrary but consistent intensity threshold.

\begin{tabular}{ccccc}
\hline & \multicolumn{4}{c}{ Cutoff $\mathrm{A}_{\mathrm{b}}$ in Pixels } \\
\cline { 2 - 5 } & 166 & 169 & 170 & 171 \\
\hline False $-\mathrm{ve}$ & $5.0 \%$ & $2.0 \%$ & $1.0 \%$ & $0.10 \%$ \\
False +ve & $21.5 \%$ & $22.0 \%$ & $22.1 \%$ & $22.2 \%$ \\
\hline
\end{tabular}

cavitation threshold empirically increases as the log of the fluid viscosity. Since the viscosities of serous and mucinous fluids are several orders of magnitude different from one another, this change in threshold should easily distinguish between the two. Cavitation may be produced in the cyst by a focused transducer under ultrasound image guidance, ideally using frequencies below diagnostic, and the increase in scatter due to bubble formation would be immediately and easily detected by diagnostic ultrasound imaging. To answer the fundamental question of serous vs. mucinous, there is no need to establish the actual threshold for a given cyst. It is enough to determine a power at which a detected cavitation signal infers the cyst is almost certainly serous, while the lack of cavitation signal is merely suggestive of a mucinous cyst. Given that the viscosity of serous fluid is below the 5\% gel and that the viscosity of mucinous fluids is above $30 \%$ gel used here based on our results, it should be possible to find an acoustic power that clearly and safely differentiates the two conditions. The outcome of our unfocused ultrasound test clearly illustrates how this might work. In that case the lowest intensity setting $\left(0.19 \mathrm{~W} / \mathrm{cm}^{2}\right)$ easily induced inertial cavitation in the samples with viscosities of less than $3 \mathrm{cP}$, yet an order of magnitude higher power $\left(2.2 \mathrm{~W} / \mathrm{cm}^{2}\right.$ calibrated output $)$ was unable to produce cavitation in the sample 
with $6600 \mathrm{cP}$ viscosity, even after multiple minutes of sonication. The lack of cavitation is a clear and unambiguous indication of the higher viscosity. While clearly an idealized model far from in vivo realization, the result suggests that this effect could be a quite robust signal for separating out a majority of patients with benign serous cysts. The remainder would then undergo a follow-up biopsy to determine the nature of their neoplasm, in line with current practice.

The approach described here would require us to overcome a few technical hurdles, such as getting focused ultrasound reliably placed in the interior of the cyst under ultrasound guidance, as well as physiological barriers. Foremost among the latter are issues of heterogeneity in cysts from patient to patient. For this technique to work, in particular, the dissolved gas content must be similar from one cyst type to another. We can surmise that this should be true based on physiological arguments, but it is not known for certain and a significant variability in this number could severely compromise the utility of our technique. If dissolved gas content is too low, it might prove difficult to safely achieve inertial cavitation in the low viscosity fluid. The fact that extracorporeal clinical focused ultrasound devices are right now capable of producing cavitation in solid tissue via high intensity pulses [19] strongly suggests that this should not be a fundamental limitation. Nevertheless, the practical aspects of issue must be assessed with further ex vivo and in vivo experiments. Another problem that will certainly arise will be differences in anatomy, resulting in difficulty accurately predicting the acoustic power actually reaching a cyst after passing through intervening layers of tissue. A simple correction with distance traveled might be sufficient, or some sort of loss might be assessed from tissues based on scattering properties measured during ultrasonic imaging. Another option might be to use acoustic radiation force inducing displacement in nearby known solid tissue to estimate the acoustic power being applied to the cyst. For these reasons, the ultrasonic cavitation thresholds reported here for our model cysts form only a starting point for in vivo efforts, which will ultimately require significant optimization.

In our proposed technique both thermal and cavitational bioeffects may be more pronounced than for regular diagnostic ultrasound, since it relies on the use of longer pulses and higher acoustic pressures. Cavitation, particularly inertial cavitation, is generally considered as an unsafe condition that is to be avoided in diagnostic ultrasound [20]-[22], but here cavitation is deliberately induced. In fact, controlled interaction with microbubbles is commonly used in imaging with ultrasound contrast agents, and the heightened risks associated with the potential development of inertial cavitation in those cases are considered acceptable [23]. Thermal effects similarly may become important where the average intensity or tissue absorption is high, but may be managed by appropriate beam forming, pulsing and targeting, as has been done clinically with MRI-guided focused ultrasound surgery, even through the skull [24] [25]. In our case, we will be dealing with power levels high enough to induce cavitation in fluid filled cavities and possibly heating near bone. However, we believe the risks can be minimized. First, the cavitation inducing beam need only to be strong enough and long enough to induce cavitation in a plasma-like fluid at the focus. This means that away from the focus and in the surrounding solid tissue the intensity should remain below the cavitation threshold under normal conditions. Second, the beam can be accurately placed inside the cyst under ultrasound image guidance. This modality can also be used to check for sensitive structures like arteries or bone in both the near and far fields prior to turning on the focused beam to avoid extraneous heating. Even if high intensities are needed, cavitation can be induced using short bursts and long duty cycle that deposit little heat in the tissue. Finally, as detailed above, since there is no need to actually induce cavitation to make a firm diagnosis, the power applied will remain strictly limited. It is anticipated that this cut-off will be sufficiently low as to preventing any heating or cavitation outside of the volume of the cyst, and that cavitation inside that volume will occur only for benign serous cysts.

Our results using both focused and unfocused ultrasound suggest that cavitation threshold can be used noninvasively to distinguish between high viscosity and low viscosity fluids in cysts, and in distinguishing mucinous vs. serous cysts in the pancreas, could potentially reduce needless biopsies on serous cysts by almost $80 \%$.

\section{References}

[1] Spence, R.A.J., Dasari, B., Love, M., Kelly, B. and Taylor, M. (2011) Overview of the Investigation and Management of Cystic Neoplasms of the Pancreas. Digestive Surgery, 28, 386-397. http://dx.doi.org/10.1159/000334758

[2] Scoazec, J.Y., Vullierme, M.P., Barthet, M., Gonzalez, J.M. and Sauvanet, A. (2013) Cystic and Ductal Tumors of the Pancreas: Diagnosis and Management. Journal of Visceral Surgery, 150, 69-84. http://dx.doi.org/10.1016/i.jviscsurg.2013.02.003

[3] Kwon, R.S. (2012) Advances in the Diagnosis of Cystic Neoplasms of the Pancreas. Current Opinions in Gastroenter- 
ology, 28, 494-500. http://dx.doi.org/10.1097/MOG.0b013e3283567f3f

[4] Eastham, R.D. (1954) The Serum Viscosity and the Serum Proteins. Journal of Clinical Pathology, 7, 66-68. http://dx.doi.org/10.1136/jcp.7.1.66

[5] Rosenson, R.S., McCormick, A. and Uretz, E.F. (1996) Distribution of Blood Viscosity Values and Biochemical Correlates in Healthy Adults. Clinical Chemistry, 42, 1189-1195. http://www.clinchem.org/content/42/8/1189.long

[6] Allen, J.S., Roy, R.A. and Church, C.C. (1997) On the Role of Shear Viscosity in Mediating Inertial Cavitation from Short-Pulse Megahertz-Frequency Ultrasound. IEEE Transactions on Ultrasound, Ferroelectrics and Frequency Control. 44, 743-751. http://dx.doi.org/10.1109/58.655189

[7] Lai, S.K., Wang, Y.Y., Cone, R., Wirtz, D. and Hanes, J. (2009) Altering Mucus Rheology to "Solidify" Human Mucus at the Nanoscale. PLoS One, 4, e4294. http://dx.doi.org/10.1371/journal.pone.0004294

[8] Lai, S.K., Wang, Y.Y., Wirtz, D. and Hanes, J. (2009) Micro- and Macrorheology of Mucus. Advanced Drug Delivery Reviews, 61, 86-100. http://dx.doi.org/10.1016/j.addr.2008.09.012

[9] Soo, M.S., Ghate, S.V., Baker, J.A., Rosen, E.L., Walsh, R., Warwick, B.N., et al. (2006) Streaming Detection for Evaluation of Indeterminate Sonographic Breast Masses: A Pilot Study. American Journal of Roentgenology, 186, 1335-1341. http://dx.doi.org/10.2214/AJR.05.0005

[10] Clarke, L., Edwards, A. and Graham, E. (2004) Acoustic Streaming: An in Vitro Study. Ultrasound in Medicine and Biology, 30, 559-562. http://dx.doi.org/10.1016/j.ultrasmedbio.2004.01.007

[11] Clarke, L., Edwards, A. and Pollard, K. (2005) Acoustic Streaming in Ovarian Cysts. Journal of Ultrasound in Medicine, 24, 617-621.

[12] Van Holsbeke, C., Zhang, J., Van Belle, V., Paladini, D., Guerriero, S., Czekierdowski, A., et al. (2010) Acoustic Streaming Cannot Discriminate Reliably between Endometriomas and Other Types of Adnexal Lesion: A Multicenter Study of 633 Adnexal Masses. Ultrasound in Obstetrics and Gynecology, 35, 349-353. http://dx.doi.org/10.1002/uog.7537

[13] Holland, C.K. and Apfel, R.E. (1989) Improved Theory for the Prediction of Microcavitation Thresholds. IEEE Transactions on Ultrasound, Ferroelectrics and Frequency Control, 36, 204-208. http://dx.doi.org/10.1109/58.19152

[14] Yang, X. and Church, C.C. (2005) A Model for the Dynamics of Gas Bubbles in Soft Tissue. Journal of the Acoustic Society of America, 118, 3595-3606. http://dx.doi.org/10.1121/1.2118307

[15] R Development Core Team (2010) R: A Language and Environment for Statistical Computing. R Foundation for Statistical Computing, Vienna. http://www.R-project.org

[16] Hyndman, R. and Fan, Y. (1996) Sample Quantiles in Statistical Packages. The American Statistician, 50, $361-365$.

[17] Lewandrowski, K.B., Southern, J.F., Pins, M.R., Compton, C.C. and Warshaw, A.L. (1993) Cyst Fluid Analysis in the Differential Diagnosis of Pancreatic Cysts. A Comparison of Pseudocysts, Serous Cystadenomas, Mucinous Cystic Neoplasms, and Mucinous Cystadenocarcinoma. Annals of Surgery, 217, 41-47. http://dx.doi.org/10.1097/00000658-199301000-00008

[18] Leung, K.K., Ross, W.A., Evans, D., Fleming, J., Lin, E., Tamm, E.P., et al. (2009) Pancreatic Cystic Neoplasm: The Role of Cyst Morphology, Cyst Fluid Analysis, and Expectant Management. Annals of Surgical Oncology, 16, 28182824. http://dx.doi.org/10.1245/s10434-009-0502-9

[19] Sokka, S.D., King, R. and Hynynen, K. (2003) MRI-Guided Gas Bubble Enhanced Ultrasound Heating in in Vivo Rabbit Thigh. Physics in Medicine and Biology, 48, 223-241. http://dx.doi.org/10.1088/0031-9155/48/2/306

[20] Shankar, H. and Pagel, P.S. (2011) Potential Adverse Ultrasound-Related Biological Effects: A Critical Review. Anesthesiology, 115, 1109-1124. http://dx.doi.org/10.1097/ALN.0b013e31822fd1f1

[21] Duck, F.A. (2008) Hazards, Risks and Safety of Diagnostic Ultrasound. Medical Engineering and Physics, 30, 13381348. http://dx.doi.org/10.1016/j.medengphy.2008.06.002

[22] O’Brien Jr., W.D. (2007) Ultrasound-Biophysics Mechanisms. Progress in Biophysics and Molecular Biology, 93, 212-255. http://dx.doi.org/10.1016/j.pbiomolbio.2006.07.010

[23] Abdelmoneim, S.S., Bernier, M., Scott, C.G., Dhoble, A., Ness, S.A.C., Hagen, M.E., et al. (2009) Safety of Contrast Agent Use during Stress Echocardiography: A 4-Year Experience from a Single-Center Cohort Study of 26, $774 \mathrm{~Pa}$ tients. JACC Cardiovascular Imaging, 2, 1048-1056. http://dx.doi.org/10.1016/j.jcmg.2009.03.020

[24] McDannold, N., Tempany, C.M., Fennessy, F.M., So, M.J., Rybicki, F.J., Stewart, E.A., et al. (2006) Uterine Leiomyomas: MR Imaging-Based Thermometry and Thermal Dosimetry during Focused Ultrasound Thermal Ablation. Radiology, 240, 263-272. http://dx.doi.org/10.1148/radiol.2401050717

[25] Lipsman, N., Schwartz, M.L., Huang, Y., Lee, L., Sankar, T., Chapman, M., et al. (2013) MR-Guided Focused Ultrasound Thalamotomy for Essential Tremor: A Proof-of-Concept Study. Lancet Neurology, 12, 462-468.

http://dx.doi.org/10.1016/S1474-4422(13)70048-6 
Scientific Research Publishing (SCIRP) is one of the largest Open Access journal publishers. It is currently publishing more than 200 open access, online, peer-reviewed journals covering a wide range of academic disciplines. SCIRP serves the worldwide academic communities and contributes to the progress and application of science with its publication.

Other selected journals from SCIRP are listed as below. Submit your manuscript to us via either submit@scirp.org or Online Submission Portal.
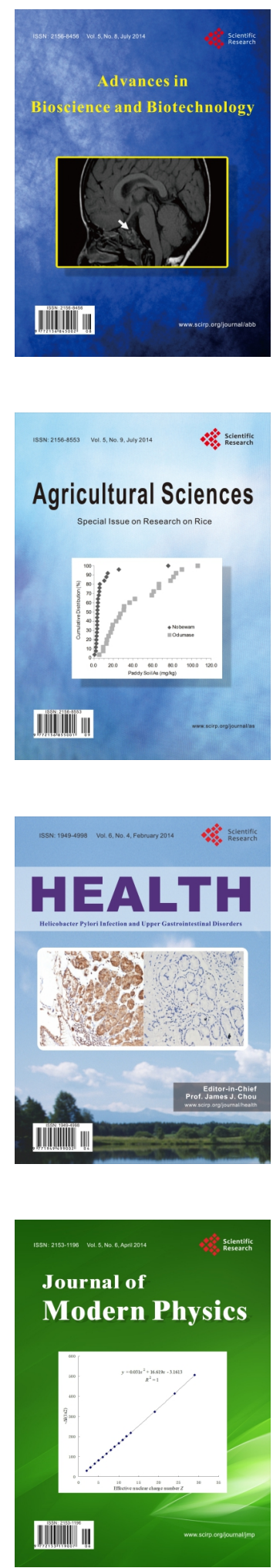
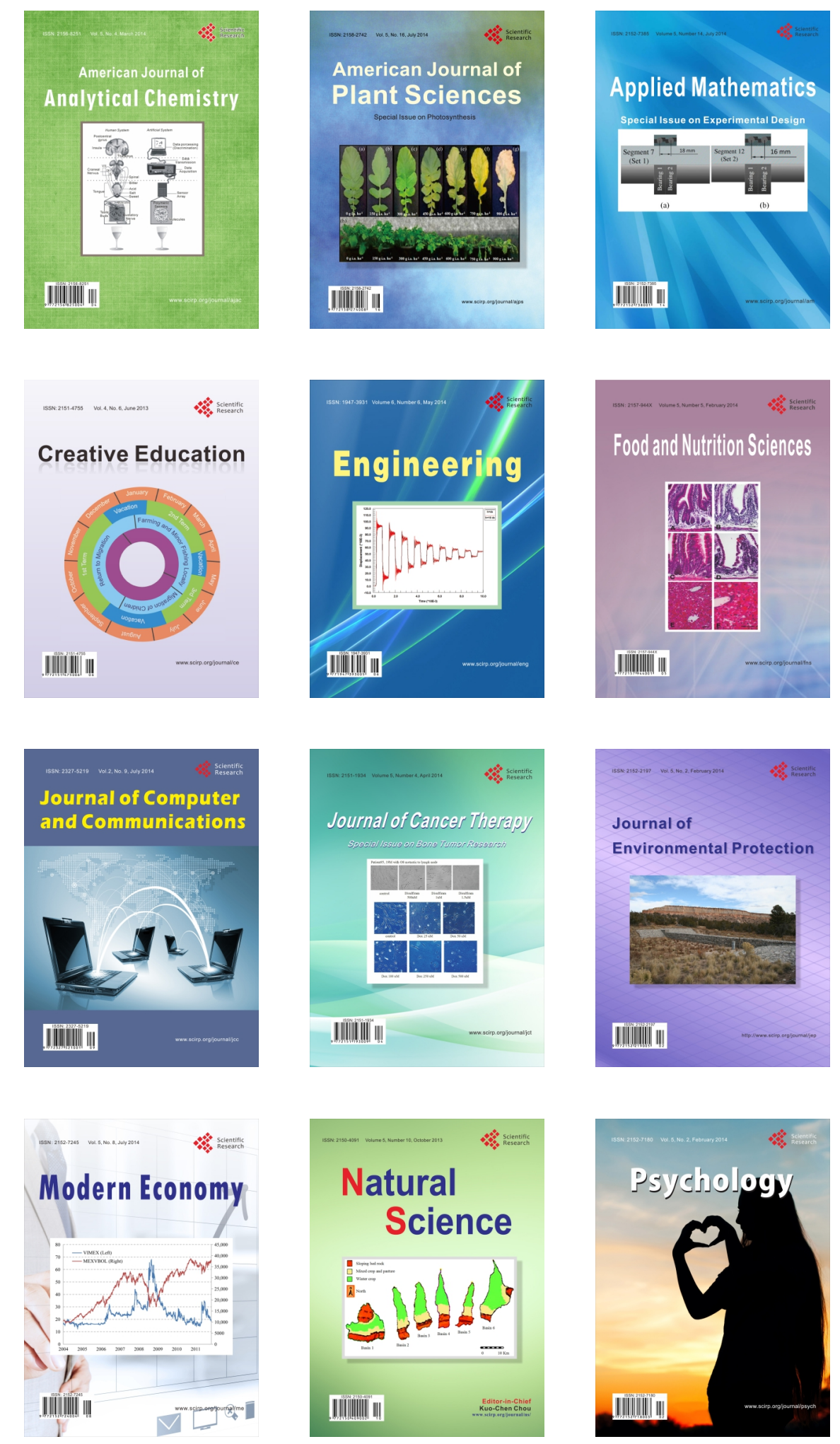